\title{
Special issue: Soil-structure interaction of buried structures
}

\author{
Guest Editor
}

Hany El Naggar, Professor, Department of Civil and Resource Engineering, Dalhousie University, Halifax, NS B3H 4R2, Canada. 